\title{
Stable chaos in fluctuation driven neural circuits
}

\author{
David Angulo-Garcia ${ }^{\mathrm{a}, *}$, Alessandro Torcinia ${ }^{\mathrm{a}, \mathrm{b}}$ \\ ${ }^{a}$ CNR - Consiglio Nazionale delle Ricerche - Istituto dei Sistemi Complessi, via Madonna \\ del Piano 10, I-50019 Sesto Fiorentino, Italy \\ ${ }^{b}$ INFN Sez. Firenze, via Sansone, 1 - I-50019 Sesto Fiorentino, Italy
}

\begin{abstract}
We study the dynamical stability of pulse coupled networks of leaky integrateand-fire neurons against infinitesimal and finite perturbations. In particular, we compare current versus fluctuations driven networks, the former (latter) is realized by considering purely excitatory (inhibitory) sparse neural circuits. In the excitatory case the instabilities of the system can be completely captured by an usual linear stability (Lyapunov) analysis, on the other hand the inhibitory networks can display the coexistence of linear and nonlinear instabilities. The nonlinear effects are associated to finite amplitude instabilities, which have been characterized in terms of suitable indicators. For inhibitory coupling one observes a transition from chaotic to non chaotic dynamics by decreasing the pulse width. For sufficiently fast synapses the system, despite showing an erratic evolution, is linearly stable, thus representing a prototypical example of Stable Chaos.
\end{abstract}

Keywords: Pulse Coupled Neural Networks, Leaky Integrate-and-Fire Model, Linear Stability Analysis, Nonlinear Stability Analysis, Lyapunov Analysis, Stable Chaos, Finite Size Lyapunov Exponent

\footnotetext{
* Corresponding author at: CNR - Consiglio Nazionale delle Ricerche - Istituto dei Sistemi Complessi, via Madonna del Piano 10, I-50019 Sesto Fiorentino, Italy. Tel.: +39 055522 6678 .

Email addresses: david.angulo@fi.isc.cnr.it (David Angulo-Garcia), alessandro.torcini@cnr.it (Alessandro Torcini)
}

Preprint submitted to Chaos, Solitons and Fractals

October 2, 2018 


\section{Introduction}

It is known that cortical neurons in vivo present a high discharge variability, even if stimulated by current injection, in comparison with neurons in vitro 1, 2]. In particular, these differences are peculiar of pyramidal neurons, while inter-neurons reveal a high neuronal firing variability in both settings [3]. This variability is usually measured in terms of the coefficient of variation $C V$ of the single neuron inter-spike interval (ISI), defined as the normalized standard deviation of the ISI, i.e, $C V=S T D(I S I) /\langle I S I\rangle$ [4]. For cortical pyramidal neurons $C V \simeq 1.0$ in vivo [1] and $C V<0.3$ in vitro [2], while for cortical inter-neurons $C V \simeq 1.0-1.2$ [3] in both settings. The variability of the spike emissions in vivo resembles a stochastic (Poissonian) process (where $C V=1$ ), however the neural dynamics features cannot be accounted by simple stochastic models [1]. These phenomena can be instead modelized by considering a deterministically balanced network, where inhibitory and excitatory activity on average compensate one another [5, 6, 7, 8]. Despite the many papers devoted in the last two decades to this subject, is still unclear which is the dynamical phenomenon responsible for the observed irregular dynamics [9, 10, 11, 12 .

A few authors pointed out the possibility that Stable Chaos |13| could be intimately related to the dynamical behavior of balanced states [14, 15, 16, 17, 18, 19]. Stable Chaos is a dynamical regime characterized by linear stability (i.e. the maximal Lyapunov exponent is negative), yet displaying an erratic behavior over time scales diverging exponentially with the system size. Stable Chaos has been discovered in arrays of diffusively coupled discontinuous maps [20] and later observed also in inhibitory neural networks [14]. This phenomenon is due to the prevalence of nonlinear instabilities over the linear (stable) evolution of the system. This leads in diffusively coupled systems to propagation of information (driven by nonlinear effects) and in diluted inhibitory networks to abrupt changes in the firing order of the neurons [13].

Clear evidences of Stable Chaos have been reported in inhibitory $\delta$-coupled networks by considering conductance based models [14] as well as current based 
models with time delay [15, 16, 17, 18]. In particular, these analysis focused on the characterization of the time needed for the transient irregular dynamics to relax to the final stable state, the authors convincingly show that these transients diverge exponentially with the system size, a key feature of Stable Chaos. Furthermore, in [16, 17] it has been shown that, by considering time extended post-synaptic pulses leads to a transition from stable to regular chaos, where fluctuation driven dynamics is apparently maintained [17].

In this paper, we would like to compare the dynamics of a balanced network, whose dynamics is driven by fluctuations in the synaptic inputs, with neural networks composed of tonically firing neurons. Similar comparisons have been performed in several previous studies [21, 22], however here we would like to focus on the role of nonlinear instabilities and in particular on indicators capable to measure finite amplitude instabilities in such networks. The effect of finite perturbations is relevant from the point of view of neuroscience, where the analysis is usually performed at the level of spike trains, and a minimal perturbation corresponds to the removal or addition of a spike. This kind of perturbations can produce a detectable modification of the firing rate in vivo in the rat barrel cortex [23]. This has been reported as the first experimental demonstration of the sensitivity of an intact network to perturbations in vivo, or equivalently of an erratic behavior in neural circuits. It is however unclear if this sensitivity should be associated to linear or nonlinear effects. In particular the authors in 23] considered a network composed of excitatory and inhibitory neurons, where an extra spike in the excitatory network is soon compensated by an extra spike in the inhibitory network, indicating a sort of balance in the activity of the studied neural circuit. The ability of a perturbed balanced network to restore rapidly the steady firing rate has been discussed also in [19] for a minimal model. Furthermore, Zillmer et al. [16] have shown that a finite perturbation in a stable regime can cause a divergence of the trajectories. These further studies, together with the fact that the addition of an extra spike is clearly a finite perturbation from the point of view of dynamical systems, suggest that the results reported in 23] can represent an experimental verification 
of Stable Chaos.

Even though all these findings are congruent with the nature of Stable Chaos [13], a careful characterization of this regime in neural networks in terms of finite amplitude indicators is still lacking. The only previous study examining this aspect in some details concerns a purely inhibitory recurrent Leaky Integrateand-Fire (LIF) neural network with an external excitatory drive, which can sustain balanced activity [19]. Starting from this analysis, which was limited to $\delta$-pulses, we have considered an extension the model to finite width pulses. Furthermore, we have characterized the linearized evolution via usual Lyapunov exponents and the nonlinear effects in terms of the response of the system to finite perturbations. This analysis has been performed by employing previously introduced indicators, like Finite Size Lyapunov Exponents (FSLEs) [24] or the probability that a finite perturbation can be (exponentially) expanded [19], and new indicators capable to capture nonlinear instabilities.

The paper is organized as follows: Section 2 is devoted to the introduction of the neural network model used through this paper, together with the indicators able to characterize linear and nonlinear instabilities. Section 3 presents a comparative study of the linear and nonlinear stability analysis with emphasis on the influence of the pulse-width and of the size of the network on the dynamical behavior. Finally, in Sect. 4 we discuss our results with respect to the existing literature and we report possible future developments of our research.

\section{Model and methods}

We will consider a network of $N$ Leaky Integrate-and-Fire (LIF) neurons, where the membrane potential $v_{i}$ of the $i$-th neuron evolves as

$$
\dot{v}_{i}(t)=a-v_{i}(t)+I_{i}(t) \quad i=1, \cdots, N \quad,
$$

where $a>1$ is the supra-threshold neuronal excitability, and $I_{i}$ represents the synaptic current due to the pre-synaptic neurons projecting on the neuron $i$. Whenever a cell reaches the threshold value $v_{t h}=1$ a pulse is emitted instantaneously towards all the post-synaptic neurons, and its potential is reset 
to $v_{r}=0$. The synaptic current $I_{i}(t)=g E_{i}$ is the superposition of the presynaptic pulses $s(t)$ received by the neuron $i$ with synaptic strength $g$, therefore the expression of the field $E_{i}$ reads as

$$
E_{i}(t)=\frac{1}{K^{\gamma}} \sum_{j \neq i} \sum_{n \mid t_{n}<t} C_{i j} \Theta\left(t-t_{n}\right) s\left(t-t_{n}\right)
$$

Where the sum extends to all the spikes emitted in the past in the network, $\Theta\left(t-t_{n}\right)$ is the Heaviside function and the parameter $\gamma$ controls the scaling of the normalization factor with the number $K$ of pre-synaptic neurons. Proper normalization ensures homeostatic synaptic inputs [25, 26]. The elements of the $N \times N$ connectivity matrix $C_{i j}$ are one (zero) in presence (absence) of a connection from the pre-synaptic $j$-th neuron to the post-synaptic $i$-th one. In this paper we limit our analysis to random sparse networks, where each neuron receives exactly $K$ pre-synaptic connections and this number remains fixed for any system size $N$. The model appearing in Eqs. (11) and (2) is adimensional, the transformation to physical units is discussed in Appendix I.

By following [5] , we assume that the pulses are $\alpha$-functions, $s(t)=\alpha^{2} t \exp (-\alpha t)$, in this case the dynamical evolution of the fields $E_{i}(t)$ is ruled by the following second order differential equation (ODE):

$$
\ddot{E}_{i}(t)+2 \alpha \dot{E}_{i}(t)+\alpha^{2} E_{i}(t)=\frac{\alpha^{2}}{K^{\gamma}} \sum_{j \neq i} \sum_{n \mid t_{n}<t} C_{i j} \delta\left(t-t_{n}\right)
$$

which can be conveniently rewritten as two first ODEs, as

$$
\dot{E}_{i}=P_{i}-\alpha E_{i}, \quad \dot{P}_{i}=-\alpha P_{i}+\frac{\alpha^{2}}{K^{\gamma}} \sum_{j \neq i} \sum_{n \mid t_{n}<t} C_{i j} \delta\left(t-t_{n}\right)
$$

by introducing the auxiliary field $P_{i}=\dot{E}_{i}-\alpha E$.

The equations (11) and (41) can be exactly integrated from the time $t=t_{n}$, just after the deliver of the $n$-th pulse, to time $t=t_{n+1}$ corresponding to the emission of the $(n+1)$-th spike, thus obtaining an event driven map [27, 28] 
which reads as

$$
\begin{aligned}
& E_{i}(n+1)=E_{i}(n) \mathrm{e}^{-\alpha \tau(n)}+P_{i}(n) \tau(n) \mathrm{e}^{-\alpha \tau(n)} \\
& P_{i}(n+1)=P_{i}(n) e^{-\alpha \tau(n)}+C_{i m} \frac{\alpha^{2}}{K^{\gamma}} \\
& v_{i}(n+1)=v_{i}(n) e^{-\tau(n)}+a\left(1-e^{-\tau(n)}\right)+g H_{i}(n),
\end{aligned}
$$

where $\tau(n)=t_{n+1}-t_{n}$ is the inter-spike interval associated to two successive neuronal firing in the network, which can be determined by solving the transcendental equation

$$
\tau(n)=\ln \left[\frac{a-v_{m}(n)}{a+g H_{m}(n)-1}\right],
$$

here $m$ identifies the neuron which will fire at time $t_{n+1}$ by reaching the threshold value $v_{m}(n+1)=1$.

The explicit expression for $H_{i}(n)$ appearing in equations (5c) and (66) is

$$
H_{i}(n)=\frac{\mathrm{e}^{-\tau(n)}-e^{-\alpha \tau(n)}}{\alpha-1}\left(E_{i}(n)+\frac{P_{i}(n)}{\alpha-1}\right)-\frac{\tau(n) e^{-\alpha \tau(n)}}{\alpha-1} P_{i}(n) .
$$

The model is now rewritten as a discrete-time map with $3 N-1$ degrees of freedom, since one degree of freedom, $v_{m}(n+1)=1$, is lost due to the event driven procedure, which corresponds to perform a Poincaré section any time a neuron fires.

Our analysis will be devoted to the study of sparse networks, by considering a constant number $K$ of afferent synapses for each neuron, namely $K=20$. Therefore, the normalization factor $K^{\gamma}$ appearing in the definition of the pulse amplitude is somehow irrelevant, since here we limit to study a specific value of the in-degree connectivity, without varying $K$. However, to compare with previous studies, we set $\gamma=1$ for purely excitatory neurons, where $g>0$, similarly to what done in [29, 30], and $\gamma=1 / 2$ for purely inhibitory networks, where $g<0$, following the normalization employed in [15, 17, 31, 19]. The reasons for these different scalings rely on the fact that in the excitatory case, the dynamics of the system are current driven (i.e. all neurons are tonically firing even in absence of coupling, being supra-threshold), therefore the synaptic input should be normalized with the number of afferent neurons to maintain an average homeostatic 
synaptic input [25, 26]. The situation is different in presence of inhibitory coupling, here the supra-threshold excitability of the single neuron can be balanced by the inhibitory synaptic currents, thus maintaining the neurons in proximity of the firing threshold. In this case, the network dynamics is fluctuation driven, because the fluctuations in the synaptic inputs are responsible of the neuronal firing. In order to keep the amplitude of the fluctuations of the synaptic current constant, the normalization is now assumed proportional to the square root of the number of the synaptic inputs [11]. In the present analysis we have tuned the model parameters in order to be in a fluctuation driven regime whenever the inhibitory coupling is considered. In particular, we will study, not only the dependence of the dynamics on the pulse shape, but also on the system size, by maintaining a constant number of incoming connections $K$. However, we will not assume that the excitatory external drive (in our case represented by the neuronal excitability a) will diverge proportionally to $\sqrt{K}$, as done in [32, 19], since we are not interested in the emergence of a self-tuned balanced state in the limit $K \rightarrow \infty$, for $1<<K<<$ [32, 19].

\subsection{Linear Stability Analysis}

To perform the linear stability analysis of the system, we follow the evolution of an infinitesimal perturbation in the tangent space, through the following set of equations obtained from the linearization of the event driven map (5a $5 \mathrm{~b}[5 \mathrm{c}$ )

$$
\begin{aligned}
\delta E_{i}(n+1) & =e^{-\alpha \tau(n)}\left[\delta E_{i}(n)+\tau(n) \delta P_{i}(n)\right] \\
& -e^{-\alpha \tau(n)}\left[\alpha E_{i}(n)+(\alpha \tau(n)-1) P_{i}(n)\right] \delta \tau(n), \\
\delta P_{i}(n+1) & =e^{-\alpha \tau(n)}\left[\delta P_{i}(n)-\alpha P_{i}(n) \delta \tau(n)\right], \\
\delta v_{i}(n+1) & =e^{-\tau(n)}\left[\delta v_{i}(n)+\left(a-v_{i}(n)\right) \delta \tau(n)\right]+g \delta H_{i}(n) \\
i & =1, \ldots, N \quad ; \quad \delta v_{m}(n+1) \equiv 0 .
\end{aligned}
$$

The boundary condition $\delta v_{m}(n+1) \equiv 0$ is a consequence of the event driven evolution. The expression of $\delta \tau(n)$ can be computed by differentiating (6) and 
(7)

$$
\delta \tau(n)=\tau_{v} \delta v_{m}(n)+\tau_{E} \delta E_{m}(n)+\tau_{P} \delta P_{m}(n),
$$

where

$$
\tau_{v}:=\frac{\partial \tau}{\partial v_{m}} \quad, \quad \tau_{E}:=\frac{\partial \tau}{\partial E_{m}} \quad, \quad \tau_{P}:=\frac{\partial \tau}{\partial P_{m}} \quad .
$$

In this paper, we will limit to measure the maximal Lyapunov exponent $\lambda$ to characterize the linear stability of the studied models. This is defined as the the average growth rate of the infinitesimal perturbation

$$
\boldsymbol{\delta}=\left(\delta v_{1} \ldots \delta v_{N}, \delta E_{1} \ldots \delta E_{N}, \delta P_{1} \ldots \delta P_{N}\right)
$$

through the equation

$$
\lambda=\lim _{t \rightarrow \infty} \frac{1}{t} \log \frac{|\boldsymbol{\delta}(t)|}{\left|\boldsymbol{\delta}_{0}\right|},
$$

where $\boldsymbol{\delta}_{0}$ is the initial perturbation at time zero. The evolution of the perturbation $\boldsymbol{\delta}(t)$ has been followed by performing at regular time intervals the rescaling of its amplitude to avoid numerical artifacts, as detailed in [33]. Furthermore, since our system is time continuous one would expect to have always a zero Lyapunov exponent, which in fact is the maximal Lyapunov if the system is not chaotic. However, this does not apply to the event driven map because the evolution is based on a discrete time dynamics, where the motion along the orbit between two successive spikes is no more present due to the performed Poincaré section.

\subsection{Finite Size Stability Analysis}

Besides the characterization of the stability of infinitesimal perturbations, we are also interested in analyzing how a perturbation grows according to its amplitude. To perform this task several indicators have been introduced in the last years, ranging from Finite Size Lyapunov Exponents (FSLE) [24, 34, 35, 36] to the propagation velocity of finite perturbations 37]. FSLEs have been mainly employed to charaterize Stable Chaos in spatially extended systems [13] and Collective Chaos in globally coupled systems [38, 39, 40]. 
We have performed several tests by employing the usual FSLE definition [36]. In particular FSLE can be defined by considering an unperturbed trajectory $\boldsymbol{x}=\left(v_{1} \ldots v_{N}, E_{1} \ldots E_{N}, P_{1} \ldots P_{N}\right)$ and a perturbed trajectory $\boldsymbol{x}^{\prime}=$ $\left(v_{1}^{\prime} \ldots v_{N}^{\prime}, E_{1}^{\prime} \ldots E_{N}^{\prime}, P_{1}^{\prime} \ldots P_{N}^{\prime}\right)$, obtained by randomly perturbing all the coordinates (both the fields $E$ and $P$ as well as the membrane potentials) of the generic configuration $\boldsymbol{x}$ on the attractor. Then we follow the two trajectories in time by measuring their distance $\Delta(t)=\left\|\boldsymbol{x}(t)-\boldsymbol{x}^{\prime}(t)\right\|$, by employing the absolute value norm. Whenever $\Delta\left(t_{k}\right)$ crosses (for the first time) a series of exponentially spaced thresholds $\theta_{k}$, where $\theta_{k}=r \theta_{k-1}$, the crossing times $t_{k}$ are registered. By averaging the time separation between consecutive crossings over different pairs of trajectories, one obtains the FSLE [36, 24]

$$
\lambda_{F}\left(\Delta\left(t_{k}\right)\right)=\frac{r}{\left\langle t_{k}-t_{k-1}\right\rangle} \quad ; \text { where } \quad \Delta\left(t_{k}\right)=\theta_{k}
$$

For small enough thresholds, one recovers the usual maximal Lyapunov exponent, while for large amplitudes, FSLE saturates to zero, since a perturbation cannot be larger than the size of the accessible phase-space. In the intermediate range, $\lambda_{F}$ tells us how the growth of a perturbation is affected by nonlinearities. However, as a general remark, we have noticed that it is extremely difficult to get reliable results from the FSLE analysis, probably because the estimation of $\lambda_{F}$ relies on measurements based on single trajectory realizations, which presents huge fluctuations. In order to overcome this problem, the single trajectory should be smoothed before estimating the passage times from one threshold to the next one and we observed that the results strongly depend on the adopted smoothing procedure, in particular for the fluctuation driven case.

Therefore, in order to investigate the growth rate of finite amplitude perturbations we have decided to adopt different indicators rather than the FSLE. In particular, an estimation of finite size stability can be obtained by defining the following indicator

$$
D(\Delta(t))=\frac{d\langle\log \Delta(t)\rangle}{d t} ;
$$

where the average $\langle\cdot\rangle$ is performed over many different initial conditions. In the limit $\Delta(t) \rightarrow 0$ we expect to recover the maximal Lyapunov exponent $\lambda$. In 
order to ensure that the dynamics of the perturbed trajectory will also occur on the attractor associated to the studied dynamics, we have considered extremely small initial perturbations $\Delta_{0}=\Delta(0) \simeq 10^{-8}-10^{-10}$. As we will show, after a transient needed for the perturbed trajectory $\boldsymbol{x}^{\prime}$ to relax to the attractor, $D(\Delta)$ measures effectively the maximal Lyapunov exponent. However, if nonlinear mechanisms are present $D(\Delta)$ can become larger than $\lambda$ for finite amplitude perturbations. Anyway, analogously to the FSLE, for perturbations of the size of the attractor the indicator $D(\Delta)$ decays towards zero due to the trajectory folding.

The studied models present discontinuities of $\mathcal{O}(1)$ in the membrane potentials $v_{i}$, due to the reset mechanisms, and of $\mathcal{O}\left(\alpha^{2} / K^{\gamma}\right)$ in the fields $P_{i}$, due to the pulse arrival. In order to reveal, without any ambiguity, the presence of nonlinear instabilities at finite amplitudes, for the estimation of the FSLE and of the indicator $D$ we mainly limit our analysis to the continuous fields $\left\{E_{i}\right\}$. In particular, to characterize the finite amplitude instabilities, we consider the following distance between the perturbed and unperturbed orbits

$$
\Delta^{(E)}(t)=\frac{1}{N} \sum_{i=1}^{N}\left|E_{i}(t)-E_{i}^{\prime}(t)\right| .
$$

In some cases we have also analyzed the distance $\Delta^{(v, E, P)}$ between all the variables associated to the unperturbed and perturbed state with a clear meaning of the adopted symbol.

Unfortunately, the indicator $D(\Delta)$ as well as the FSLE cannot be employed in the case of stable chaos, when $\lambda<0$, because in this case small perturbations are quickly damped and one cannot explore the effect of perturbation of growing amplitude by following the dynamics on the attractor. In this situation, one should employ different indicators, as done in [34, 41] for coupled map lattices. In particular, we proceed as follow, we consider two orbits at an initial distance $\Delta_{0}$ and we follow them for a time interval $T$, then we measure the amplitude of the perturbation at the final time, namely $\Delta(T)$. We rescale one of the two orbits to a distance $\Delta_{0}$ from the other one, keeping the direction of the perturbation unchanged, and we repeat the procedure several times and for several values of 
$\Delta_{0}$. Then, we estimate the finite amplitude growth rate, as

$$
R_{T}\left(\Delta_{0}\right)=\frac{1}{T}\left\langle\log \frac{|\Delta(T)|}{\left|\Delta_{0}\right|}\right\rangle,
$$

where the angular brackets denote the average over a sufficiently large number of repetitions. To allow the perturbed orbit to relax on the attractor, we initially perform $\simeq 10^{3}$ rescalings, which are not included in the final average. However, also this procedure does not guarantee that the attractor is always reached, in particular for very large perturbations. Furthermore, the perturbed dynamics is no more constrained to evolve along the tangent space associated to the event driven map. As a matter of fact, whenever $\lambda<0$ the indicator $R_{T}\left(\Delta_{0}\right)$ converges to zero and not to the Lyapunov exponent associated to the discrete time map evolution.

Finally, following the analysis reported in [19], we consider the probability $P_{S}\left(\Delta_{0}\right)$ that a perturbation of amplitude $\Delta_{0}$ induces an exponential separation between the reference and perturbed trajectory. In particular, we perturb the reference orbit with an initial perturbation $\Delta_{0}$ and we follow the evolution of the trajectories for a time span $T$. Whenever $\Delta(T)$ is larger than a certain threshold $\theta_{L}$ this trial contributes to the number of expanding initial perturbations $N_{S}\left(\Delta_{0}\right)$, otherwise is not counted. We repeat this procedure $N_{T}$ times for each perturbation of amplitude $\Delta_{0}$, then $P_{S}\left(\Delta_{0}\right)=N_{S}\left(\Delta_{0}\right) / N_{T}$. For the two latter indicators, namely $R_{T}$ and $P_{S}$, we have always employed the total distance $\Delta^{(v, E, P)}$, to confront our findings with the results reported in [19].

\section{Results}

As already mentioned, we will compare a current driven excitatory network and a fluctuation driven inhibitory network. In particular, the excitatory network is studied in a regime where it presents a collective non trivial partial synchronization [42, 30]. This state is characterized by quasi-synchronous firing events, as revealed by the raster plot reported in the upper panel of Fig. 1(a) and almost periodic oscillations of the effective current $I_{i}^{e f f}(t) \equiv a+g E_{i}(t)$ 

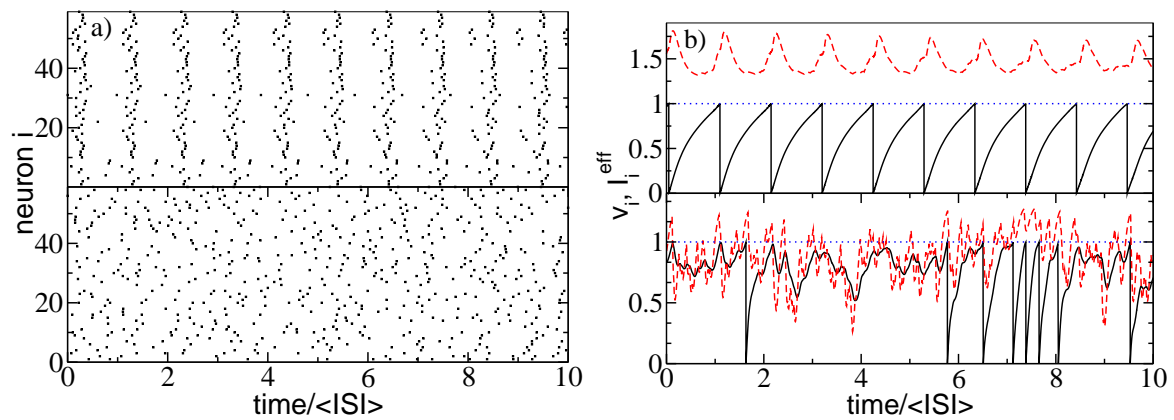

Figure 1: Comparison between current driven (upper panels) and fluctuation driven (lower panels) activity. (a) Raster plots for a pool of 60 neurons. (b) Membrane potential traces $v_{i}(t)$ (black solid line) and the corresponding effective current $I_{i}^{\text {eff }}$ (red dashed line) for a typical neuron. The blue dotted line indicates the firing threshold. For the current driven case, $a=1.3, g=0.2, \alpha=9$ and $\gamma=1$, corresponding to the situation studied in [30]; for the fluctuation driven network the parameters are the same, apart $g=-0.8, \alpha=5$ and $\gamma=1 / 2$. For both networks, $K=20$ and $N=400$ and the results for both systems are reported for the same rescaled time intervals $t /\langle I S I\rangle=10$, after discarding a transient of $10^{4}$ spikes.

(see Fig. 1(b) upper panel). In this particular case $I_{i}^{\text {eff }}>1$ therefore the neurons are always supra-threshold. In this situation the measure of the $C V$ gives quite low values, namely for the studied case (with $a=1.3, g=0.2$ and $\alpha=9) C V \simeq 0.17$, similar to pyramidal neurons in vitro. Despite this low level of variability in the neuronal dynamics, the sparseness in the matrix connectivity induces chaotic dynamics in the network, which persists even in the thermodynamic limit [30]. At variance with diluted networks, where the average connectivity scales proportionally to the system size $\left(K \propto N^{z}\right.$, with $1 \geq z>0$ ). In this latter case, in the limit $N \rightarrow \infty$ the system will recover a regular evolution, similarly to fully coupled networks [28, 29].

For the inhibitory network, we observe radically different dynamics, this because now $I^{e f f}(t)$ oscillates around one, therefore the neurons fire in a quite irregular manner, driven by the fluctuations of the fields $E_{i}(t)$, as shown in the lower panels of Figs. 1 (a) and (b). In this case we have examined the dynamics of the model for $a=1.3, g=-0.8$ and different pulse-widths $1 / \alpha$. For $\alpha \in[1: 5]$ the neuronal dynamics are always quite erratic, being characterized by 

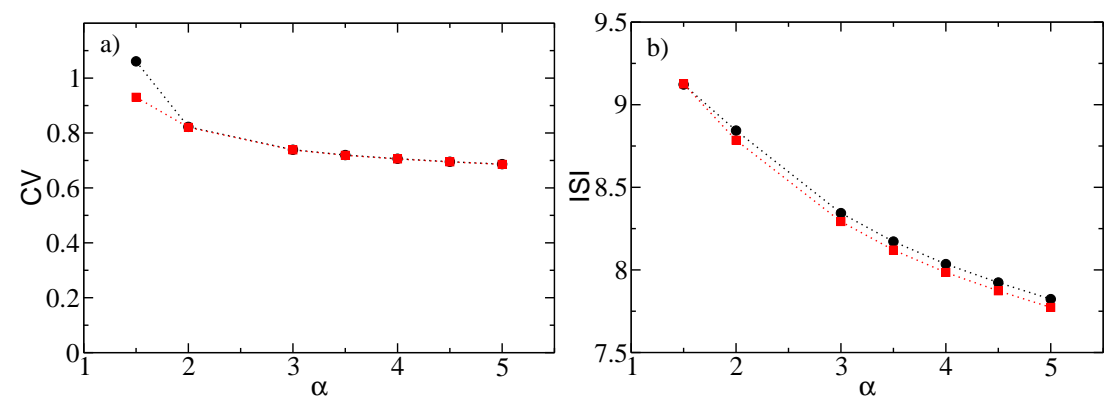

Figure 2: Dependence of the coefficient of variation $C V$ (a) and of the inter-spike time interval ISI (b) on the pulse width for the fluctuation driven case. The data refer to $N=400$ (black circles) and $N=1600$ (red squares). The data have been averaged over $10^{8}$ spikes, once a transient of $10^{7}$ spikes has been discarder. The other parameters are as in the caption of Fig. 1.

$C V \simeq 0.7-1$ (see Fig. 2(a) . Narrower pulses (larger $\alpha$ values) are associated to somehow more regular dynamics and smaller ISI, however we have verified that the ISI and $\mathrm{CV}$ saturates to some finite value in the thermodynamic limit (as shown in Fig. 3 (a) and (b)). This suggests that fluctuations will not vanish for $N \rightarrow \infty$ and that the system will remain fluctuation driven even in such a limit. Furthermore, the two $\alpha$-values examined in Figs. 3(a) and 3(b) correspond to two different dynamical regimes, further discussed in Sect. 3.1 namely, a chaotic $(\alpha=3)$ and a non-chaotic $(\alpha=5)$ state.

\subsection{Lyapunov analysis}

As previously shown, the fluctuation driven regime is observable for the inhibitory network for all the considered pulse widths. In this Subsection we would like to investigate whether such variability is related to a linear instability of infinitesimal perturbations (measured by the maximal Lyapunov exponent $\lambda$ ) or to other (nonlinear) instabilities present in the system. Let us start examining the Lyapunov exponent for such systems, as a first result we observe a strong dependence of $\lambda$ on the pulse-width (see Fig. 4(a) : the system is chaotic for wide pulses and becomes stable for sufficiently narrow ones. These results are in agreement with previously reported results in [16, 17] for an inhibitory net- 

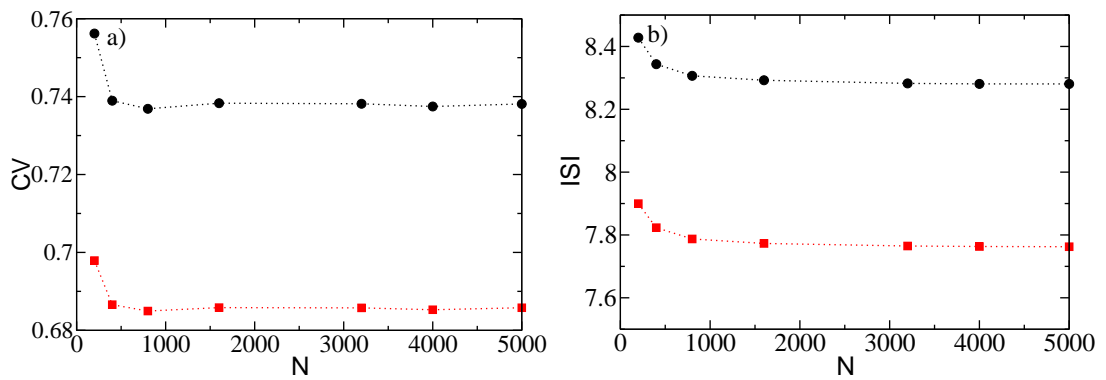

Figure 3: Dependence of the coefficient of variation $C V$ (a) and of the inter-spike time interval ISI (b) on the size of the network for fluctuation driven networks in two representative situations corresponding to the chaotic ( $\alpha=3$, black circles) and the stable chaos $(\alpha=5$, red squares) regimes. The reported data have been averaged over $10^{8}$ spikes, once a transient of $10^{7}$ spikes has been discarded. The other parameters are as in the caption of Fig. [1

work of LIF neurons with delayed synapses. In these papers the authors show that chaos can arise only for sufficiently broad pulses, conversely for $\delta$-pulses the system is always stable. It is worth to notice that the critical $\alpha$-value at which occurs the transition to chaos becomes larger as the system size increases, pointing to the question whether the stable regime still exists for finite pulses in the thermodynamic limit or if it is merely a finite size property [17]. Extensive simulations for sizes of the network up to $N=10,000$ have shown that the stable regime is present even for such a large size (see Fig 4(b). Furthermore, we have found an empirical scaling law describing the increase of $\lambda$ with $N$, i.e.

$$
\lambda=\lambda_{\infty}-c N^{-\eta}
$$

where $\lambda_{\infty}$ denotes the asymptotic value in the thermodynamic limit and $\eta$ is the scaling exponent. For the two representative cases here studied, the exponent was quite similar, namely $\eta \simeq 0.24(\eta \simeq 0.22)$ for $\alpha=3(\alpha=5)$, thus suggesting an universal scaling law for this model when fluctuation driven, with an exponent $\eta=1 / 4$. This exponent is different from the one measured for the current driven case, in such situation for sparse connectivity $\lambda$ converged to its asymptotic value as $1 / N$ [30]. An exponent $\eta=1$ has been previously measured for coupled map lattices exhibiting spatio-temporal chaos and the- 
oretically justified in the framework of the Kardar-Parisi-Zhang equation [43]. The scalings we are reporting in this paper are associated to random networks, therefore they demand for a new theoretical analysis. Furthermore, the asymptotic values $\lambda_{\infty}=0.335(1)\left(\lambda_{\infty}=-0.034(1)\right)$ indicate that a critical threshold separating stable from chaotic dynamics persists in the thermodynamic limit.
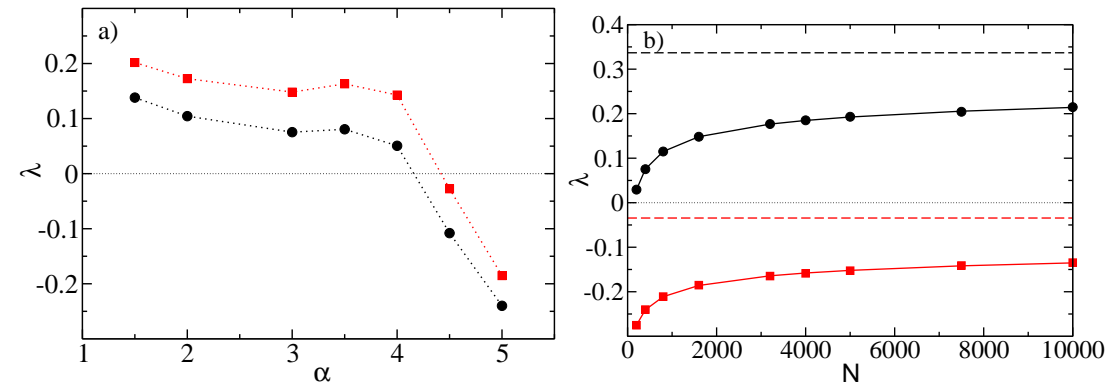

Figure 4: Linear stability analysis of the fluctuation driven state. (a) Maximal Lyapunov exponent $\lambda$ as a function of pulse-width $\alpha$, for two representative system sizes: $N=400$ (black circles) and $N=1600$ (red squares); thin dashed lines are drawn for eye guide only. (b) Lyapunov exponent as a function of the system size $N$, for two representative pulse widths: $\alpha=3$ (black circles) and $\alpha=5$ (red squares). Continuous lines correspond to the nonlinear fitting (16), which predicts the asymptotic values $\lambda_{\infty}$ (thick dashed lines). The fitting parameters entering in Eq. (16) are $c=1.08(c=0.78)$ and $\eta \simeq 0.24(\eta \simeq 0.22)$ for $\alpha=3(\alpha=5)$. In both figures, $\lambda$ is calculated by integrating the evolution in the tangent space together with the unperturbed orbit dynamics during a time interval equivalent to $10^{8}$ spikes, after discarding a transient of $10^{7}$ spikes. Remaining parameters as in Fig. 1

\subsection{Finite size perturbation analysis}

Stable chaos in spatially extended systems is due to the propagation of finite amplitude perturbations, while infinitesimal ones are damped. In inhibitory neural networks, the origin of Stable Chaos has been ascribed to abrupt changes in the firing order of neurons induced by a discontinuity in the dynamical law, while infinitesimal perturbations leave the order unchanged [14, 13, 17]. In particular, by examining a conductance based model, in [13] it has been shown that a spike was able to induce a finite perturbation in the evolution of two (not-symmetrically) connected neurons, given that the inhibitory effect of a 
spike was related to the actual value of the membrane potential of the receiving neuron. Therefore two ingredients are needed to observe Stable Chaos in neural models, a non symmetric coupling among neurons, together with the fact that the amplitude of transmitted pulses should depend on the neuron state. These requirements are fulfilled also in the present model, despite being current based, since any current based model can be easily transformed in a conductance based one via a nonlinear transformation [5, 44]. However, the problem is to quantify this effect in terms of some indicator, similarly to what done in spatially extended systems, where Stable Chaos has been characterized in terms of the FSLE and of the velocity of propagation of information [37, 41].

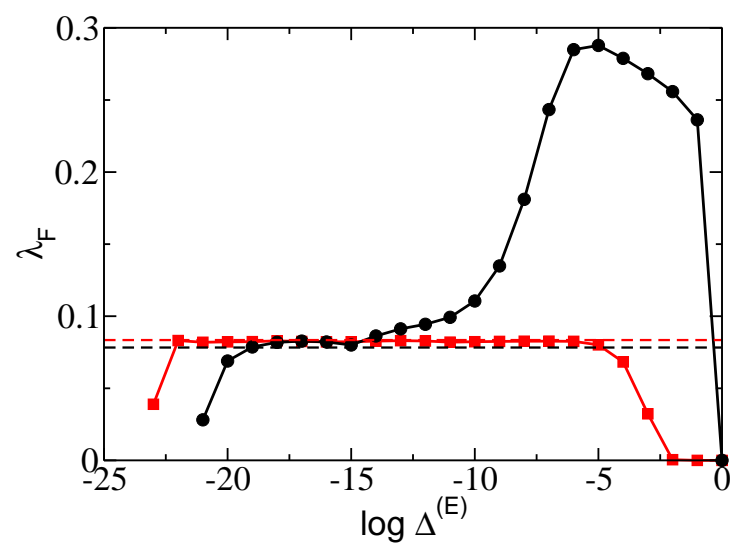

Figure 5: FSLE indicator $\lambda_{F}$ for the fluctuation driven (black circles) and current driven (red squares) chaotic set-ups. An initial perturbation of $10^{-9}\left(10^{-7}\right)$ is applied to the excitatory (inhibitory) network. The distance between the perturbed and unperturbed trajectory $\Delta^{(E)}$ is sampled during 300 time units, at fixed time intervals $d t=0.2$. The sampled curve is smoothed over a sliding window of 20 time units and the resulting curve is used to obtain the times $t_{k}$ at which the system crosses the corresponding thresholds $\theta_{k}$, with $r=1$ (see the definition (12). This procedure is averaged in the current (fluctuation) driven case over 5000 (15000) realizations. Thick dashed lines indicate the value of $\lambda$ for each one of the two cases. The current and fluctuation driven cases have been examined for the same parameter values reported in Fig. 1 apart that for the inhibitory case the inverse of the pulse width is set to $\alpha=3$.

As a first indicator we consider the FSLE, associated to the norm $\Delta^{(E)}$, the corresponding results are reported in Fig. 5 for the current and fluctuation 
driven cases. In the former case the FSLE is never larger than the usual Lyapunov exponent $\lambda$, with which it coincides over a wide range of perturbation amplitudes. In particular, $\lambda_{F}\left(\Delta^{(E)}\right)<\lambda$ for small amplitudes, due to the fact that initially the perturbation needs a finite time to align along the maximal expanding direction. Furthermore, due to the folding mechanism, the perturbation is contracted also for large perturbations of the order of the attractor system size. In summary, for current driven dynamics only the instability associated to infinitesimal perturbation is present, as reported also in [40]. In the fluctuation driven case the situation is quite different as shown in Fig. 5, the FSLE essentially coincides with $\lambda$ for small $\Delta^{(E)}$, but it becomes definitely larger than $\lambda$ for finite perturbations, revealing a peak around $\Delta^{(E)} \simeq \mathcal{O}(1 / N)$. These are clear indications that finite amplitude instabilities coexist with infinitesimal ones and they could be in principle even more relevant.

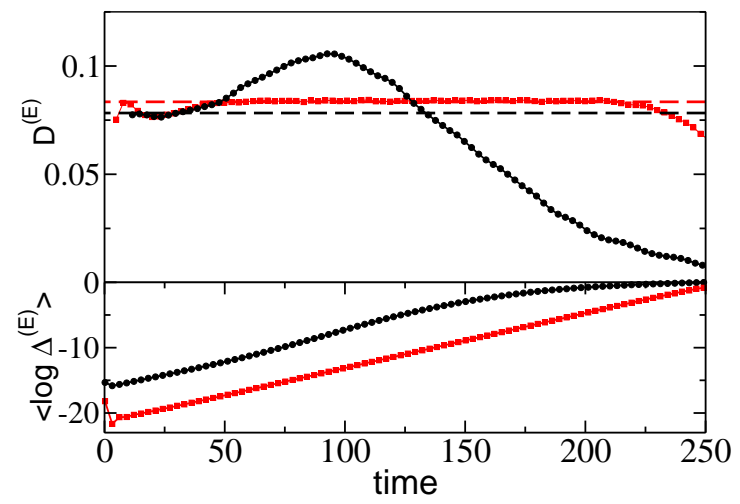

Figure 6: Lower panel: Evolution of the average distance $\left\langle\log \Delta^{(E)}\right\rangle$ as a function of time, for the current (red square) and the fluctuation (black circle) driven cases. The curves are obtained by averaging the distances between the perturbed and unperturbed trajectories over 5000 (15000) realizations, after applying an initial perturbation of $\mathcal{O}\left(10^{-8}\right)$. Upper panel: Indicator $D^{(E)}$ as a function of time for the same cases, calculated as the time derivative of $<\log \Delta^{(E)}>$. For small perturbations, $D^{(E)}$ is close to $\lambda$ (thick dashed lines), while observing a finite size effect is observable in the fluctuation driven case. The current and fluctuation driven cases have been examined for the same parameter values reported in Fig. 5

The estimation of the FSLE, as already mentioned, suffers of several numerical problems in these systems. Therefore we decided to consider the indicator 
$D\left(\Delta^{(E)}(t)\right)$, for simplicity denoted as $D^{(E)}$, which is less affected by the single orbit fluctuations, since its estimation is based on the time derivative of the averaged distance $\langle\log \Delta(t)\rangle$. In Fig. [6 we report $\left\langle\log \Delta^{(E)}(t)\right\rangle$ and $D^{(E)}$ as a function of time for a current driven and a fluctuation driven case, in both situations after an initial transient, the indicator $D^{(E)}$ coincides with $\lambda$. However, in the current driven case it coincides with $\lambda$ for a very long time before decreasing due to the folding of the trajectories, while in the fluctuation driven situation it becomes soon larger than the maximal Lyapunov exponent and it shows a clear peak at finite amplitudes, before the folding effect sets in. The same results are reported in the upper panel of Fig. 7 as a function of $\left\langle\log \Delta^{(E)}(t)\right\rangle$, the peak in the fluctuations driven case is located around $4 \times 10^{-4}$ thus at a smaller amplitude with respect to the FSLE, despite the system size and parameters are the same in both cases. Furthermore, in the lower panel in Fig. 7 we report the indicator $D\left(\Delta^{v, E, P}(t)\right)\left(D^{(v, E, P)}\right.$ from now on) estimated for the total distance among the perturbed and unperturbed orbit. As expected, the discontinuities present in the evolution of the membrane potentials and of the auxiliary field $P$ due to pulse emission and pulse arrival, induce a small increase on $D^{(v, E, P)}$ with respect to the infinitesimal value $\lambda$ at finite amplitudes even in the current driven case. However, in this case the peak of $D^{(v, E, P)}$ is definitely smaller with respect to the one observed in the fluctuation driven case and it is located at larger perturbations $\mathcal{O}(1)$. Similar effects are observable also by considering the FSLE associated to $\Delta^{(v, E, P)}$, data not shown. Nevertheless, in order to keep ourselves in a consistent framework, in what follows we will consider the distance between the perturbed and unperturbed continuous fields $\Delta^{(E)}$. By choosing this norm, we will avoid the presence of (trivial) peaks due to discontinuities as in the current driven system, but instead, the presence of these peaks will be a genuine indication of nonlinear instabilities, as those present in a fluctuation driven regime.

The indicator $D^{(E)}$ is reported in Fig. 8 for various system sizes, ranging from $N=400$ to $N=1600$ for the current and fluctuation driven cases. We observe that in the current driven case $D^{(E)}$ always gives a value around the 


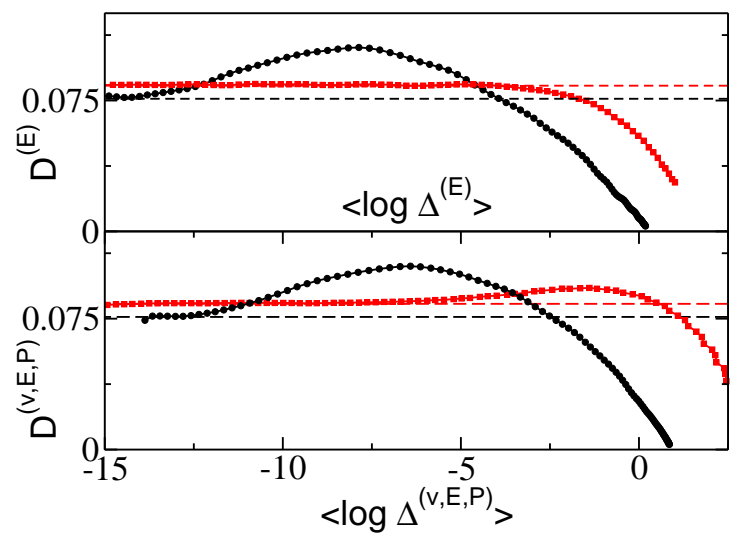

Figure 7: Indicator $D(\Delta)$ versus the complete distance $\Delta^{(v, E, P)}$ (lower panel) and versus the distance $\Delta^{(E)}$ (upper panel) for the current (red squares) and fluctuation (black circles) driven cases. The curves are obtained with the same procedure described in the caption of Fig. 6] In both panels, thick dashed lines illustrate the corresponding value of $\lambda$. The current and fluctuation driven cases have been examined for the same parameter values reported in Fig. 5]

corresponding $\lambda$ at all scales, apart the final saturation effect (see Fig. 8(a) . Notice that $\lambda$, for these system sizes, strongly depends on $N$ (as shown in [30]), the saturation at the asymptotic value is expected to occur for $N>5000$. For the fluctuation driven set-up, a peak (larger than $\lambda$ ) is always present in $D^{(E)}$ at finite amplitudes (see Fig. 8(b)]. The peak broadens for increasing $N$ extending to larger amplitudes and also its height increases. The presence of more neurons in the network renders stronger the finite amplitude effects, while nonlinear instabilities are present at larger and larger perturbation amplitudes.

So far we have considered only chaotic regimes, both in the fluctuation driven and in the current driven case. However, even in linearly stable cases the dynamics can be erratic, as shown in Fig. 1 for the fluctuation driven case corresponding to $\alpha=5$ for which the maximal Lyapunov is negative at any system

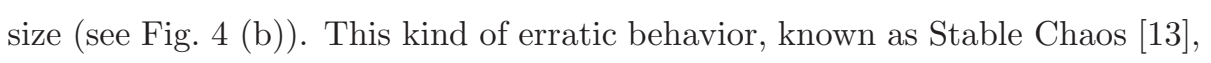
is one the most striking examples of dynamics driven by nonlinear effects, since the linear instabilities are asymptotically damped. In this situation neither the FSLE nor the indicator $D(\Delta)$ can be measured. The reason is that, in order 

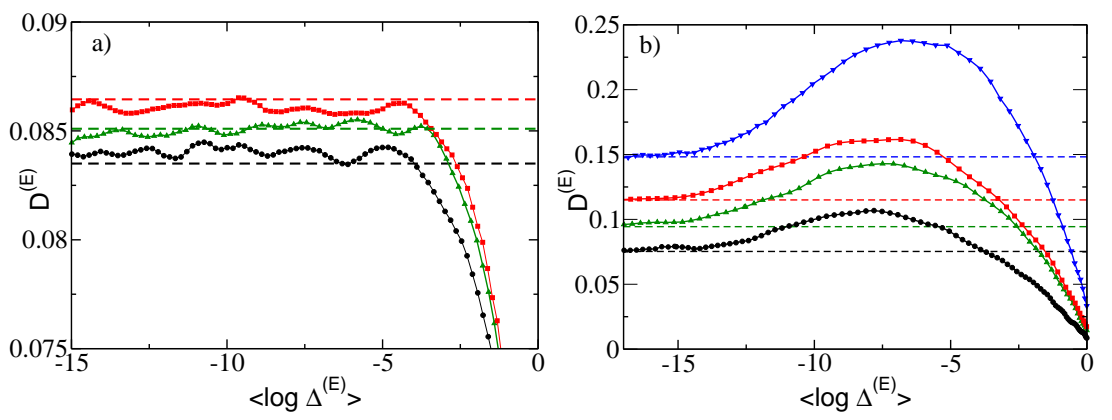

Figure 8: Finite amplitude perturbation analysis for several sizes of the network by using the procedure described in Fig. 7 for the distance $\Delta^{E}$ for the current (a) and fluctuation (b) driven setups. In both cases the studied sizes correspond to $N=400$ (black circles), $N=600$ (green up-triangles), $N=800$ (red squares) and $N=1600$ (blue down-triangles), averaged through 7500 realizations. Remaining parameters as in Fig. 5

to ensure that the dynamics will take place on the associated attractor, finite amplitude perturbations are reached only by starting from very small initial perturbations, which in this case are damped. Therefore, we should employ different indicators, namely the finite amplitude growth rate $R_{T}\left(\Delta_{0}\right)$ and the probability $P_{S}\left(\Delta_{0}\right)$.

As shown in Fig. 9(a) for the linearly stable fluctuation driven case corresponding to $\alpha=5, R_{T}\left(\Delta_{0}\right) \rightarrow 0$ for sufficiently small perturbations, as expected. However $R_{T}\left(\Delta_{0}\right)$ becomes soon positive for finite amplitude perturbation and it reveals a large peak $R_{T}^{M}$ located at an amplitude $\Delta_{0}^{M}$. For increasing system size $N$, as shown in Fig. 10(a) a linear decrease of $\Delta_{0}^{M}$ with $N$ is clearly observable, while $R_{T}^{M}$ reveals a logarithmic increase with $N$. Thus suggesting that this indicator will diverge to infinity in the thermodynamic limit, similarly to the results previously reported in [32, 19]. However, at variance with these latter studies, in the present context the connectivity remains finite even in the limit $N \rightarrow \infty$.

The analysis of $P_{S}\left(\Delta_{0}\right)$, reported in Fig. 9(b), reveals that the curve can be well fitted as

$$
P_{S}\left(\Delta_{0}\right)=1-\exp \left(-\Delta_{0} / \beta\right)^{\mu} \quad ;
$$


analogously to what done in [19]. The parameter $\beta$ can be considered as a critical amplitude, setting the scale over which nonlinear instabilities take place. At variance with the results reported by Monteforte \& Wolf in [19], we observe a linear decrease with $N$ of the critical amplitude $\beta$ (see Fig. 10(b) and an exponent $\mu \simeq 2.3-2.5$, depending on the employed system size. Instead, Monteforte \& Wolf reported a scaling $\beta \propto 1 / \sqrt{N}$ and an exponent $\mu=1$. Furthermore, we have verified for various continuous $\alpha$ pulses, with $\alpha \in[4 ; 7]$, that the measured exponent $\mu$ does not particularly depend on $\alpha$. The model here studied differs for the shape of the post-synaptic currents from the one examined in [19], where $\delta$-pulses have been considered.
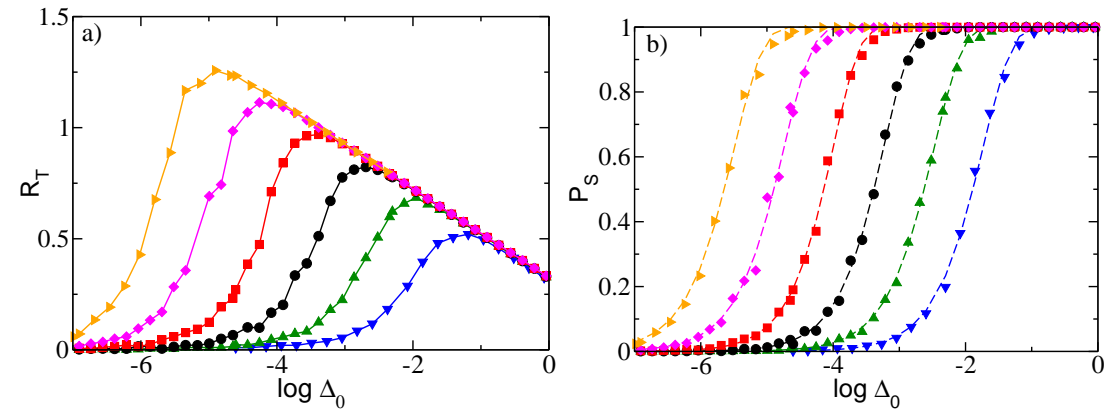

Figure 9: Characterization of the Stable Chaos regime: finite amplitude instabilities for different network sizes. (a) $R_{T}$ indicator as a function of the initial perturbation $\Delta_{0}$. (b) Probability $P_{S}$ to observe an exponential increase of the distance between a perturbed and an unperturbed orbit versus the initial perturbation $\Delta_{0}$. Thick dashed lines refer to the fit to the data with the expression $P_{S}=1-e^{-\left(\Delta_{0} / \beta\right)^{\mu}}$. The studied sizes are $N=100$ (blue down-triangle), $N=200$ (green up-triangles), $N=400$ (black circles), $N=800$ (red squares), $N=1600$ (magenta diamonds) and $N=3200$ (orange right-triangles). For each perturbation $\Delta_{0}, R_{T}$ and $P_{S}$ are calculated after $T=5$ time units, threshold defining expanding trajectories $\theta_{L}=-2$ and averaging over $N_{T}=5000$ realizations. Remaining parameters as reported in Fig. 1

In our opinion, these two latter indicators, $R_{T}$ and $P_{S}$ bear essentially the same information: they measure the propensity of a perturbation $\Delta_{0}$ to be amplified on a short time scale $T$. This is confirmed by the fact that (as shown in Fig. 10) the values of $\Delta_{0}^{M}$ and $\beta$, which set the relevant amplitude scales 
for the two indicators, both decrease with the same scaling law (namely, $1 / N$ ) with the system size. A possible explanation for this scaling could be found by assuming that the main source of nonlinear amplification is associated to a spike removal (addition) in the perturbed orbit. A missing (extra) spike will perturb, to the leading order, the distance $\Delta^{(v, E P)}$ by an amount $\propto \alpha^{2} \sqrt{K} / N$, since the lost (added) post-synaptic pulses are $K$ each of amplitude $\alpha^{2} / \sqrt{K}$. This argument explains as well the logarithmic increase of $R_{T}^{M}$ with the system size. Furthermore, the decrease of $\Delta_{0}^{M}$ and $\beta$ with $N$ seems to indicate that in the thermodynamic limit any perturbation, even infinitesimal, will be amplified. This is clearly in contradiction with the fact that the system is linearly stable and it appears to remain stable by increasing $N$ (as shown in Fig. 4(b)]. In systems exhibiting Stable Chaos, it has been reported many times the fact that the thermodynamic limit and the infinite time limit do not commute [20]. For finite system size, at sufficiently large times (diverging exponentially with $N$ ) a stable state is always recovered, while if the thermodynamic limit is taken before the infinite time one, the system will remain erratic at any time [13]. In the present case, it seems that a different non commutativity between the thermodynamic limit and the limit of vanishingly small perturbations is present, similar conclusions have been inferred also in [19]. Therefore, we can apparently conclude that a fluctuation driven system, which is linearly stable, but presents nonlinear instabilities, will become unstable at any amplitude and time scales in the thermodynamic limit. However, one should be extremely careful in deriving any conclusion from these indicators, since they are not dynamical invariant and their values depend not only on the considered variables but also on the employed norm. Furthermore, in the present context there is an additional problem related to the meaningful definition of the norm in an infinite space, as that achieved in the thermodynamic limit.

To understand the limit of applicability of $R_{T}$, we have examined this indicator also in the chaotic fluctuation driven case, namely for $\alpha=3$. Also in this case we observe that $\Delta_{0}^{M}$ will vanish for diverging system size, but with a different scaling law, namely $\Delta_{0}^{M} \simeq N^{-0.6}$. Furthermore, $R_{T}^{M}$ increases with $N$, 

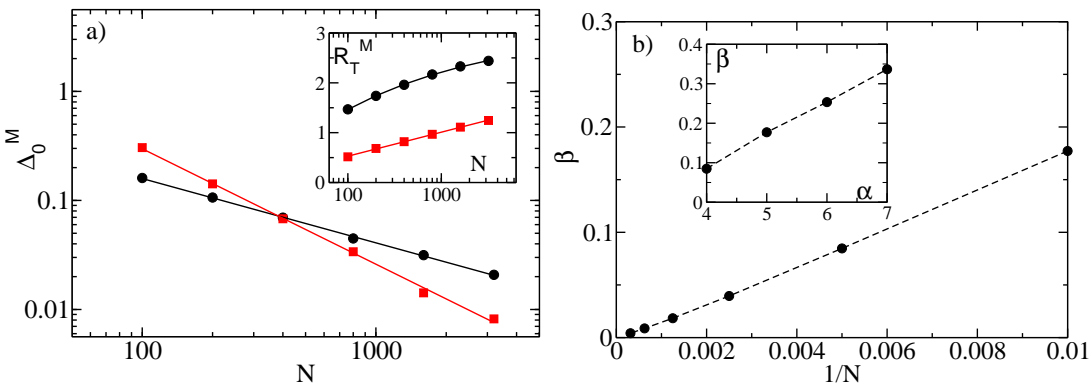

Figure 10: a) Peak position $\Delta_{0}^{M}$ as a function of $N$ in a log-log scale for $\alpha=3$ (black circles) and $\alpha=5$ (red squares). The continuous line are power law fitting $\Delta_{0}^{M} \propto N^{-\Phi}$, with exponents $\Phi=-0.59(\Phi=-1.05)$ for $\alpha=3(\alpha=5)$. Inset, maximum value of $R_{T}$ as a function of the number of neurons in the network $N$ in a $\log$-lin scale. The solid lines refer to fittings to the data, namely $R_{T}^{M}=3.09-5.60 N^{-0.27}$ for $\alpha=3 ; R_{T}^{M}=0.23+0.28 \log (N)$ for $\alpha=5$. $R_{T}$ calculated after a time span $T=1(T=5)$ for $\alpha=3(\alpha=5)$. b) Amplitude scale $\beta$ associated to the indicator $P_{S}$ as a function of $1 / N$. In the inset, $\beta$ is reported as a function of $\alpha$ for parameter values associated to non chaotic dynamics. In the same range the exponent $\mu \sim 2.32$ (not shown). The model parameters refer to the fluctuation driven case studied in Fig. 1 Inset is obtained with $N=100$

but this time it appears to saturate in the thermodynamic limit with a scaling law similar to the one reported in (16) for the maximal Lyapunov exponent, more details are reported in the caption of Fig. 10. Unlike the stable regime, in the chaotic one we cannot justify with the simple spike addition (removal) argument the scaling with $N$ neither for $\Delta_{0}^{M}$ nor for $R_{T}^{M}$. It is high probable that in this regime the interactions of the linear and nonlinear instabilities leads to more complicated mechanisms. The evolution of the indicator $R_{T}$ suggests that for increasing $N$ its peak will move down to smaller and smaller amplitude scale. However, this result is in contradiction with the behavior of $D^{(E)}$ reported in Fig. 8(b) for this latter indicator the position of the peak is not particularly affected by $N$. In particular, finite amplitude instabilities affect larger and larger scales, contrary to what seen for $R_{T}$ (see Fig. 9(a)). The same behavior is observable for $D^{(v, E, P)}$, data not shown. These contradictory results point out the limits of indicators like $R_{T}$ and $P_{S}$ relying on dynamical evolutions not taking place on the attractor of the system. 

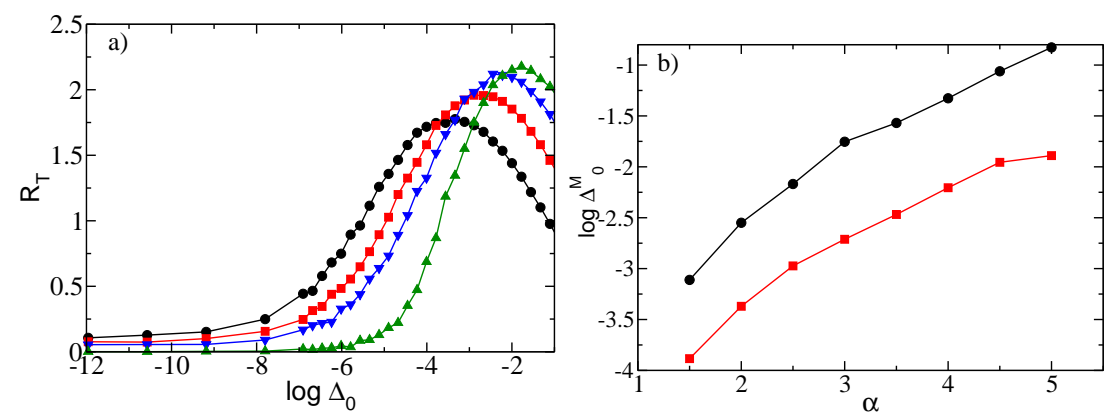

Figure 11: Finite size instabilities for fluctuation driven dynamics, for different pulse widths.

(a) $R_{T}$ as a function of the initial perturbation $\Delta_{0}$, for $\alpha=2$ (black circle), $\alpha=3$ (red square), $\alpha=4$ (blue down-triangle), $\alpha=5$ (green up-triangle). The system size is fixed to $N=400$. (b) Peak location in logarithmic scale $\log \Delta_{0}^{M}$ as a function of the inverse pulse-width $\alpha$, for two sizes of the network: $N=100$ (black circles) and $N=400$ (red squares). The peak positions were found by fitting a quadratic function around the maximum of the function $R_{T}$ in (a). $R_{T}$ is calculated as described in the caption of Fig. 9 Remaining parameters as in Fig. 1

Finally, in order to study the effect of the pulse shape on the finite amplitude behavior as measured by $R_{T}$, we proceeded to calculate this indicator for various $\alpha$-values. As shown in Fig. 11 for increasing $\alpha$ (corresponding to narrower peaks) the position of the maximum $\Delta_{0}^{M}$ moves towards larger amplitudes. This effect can be explained by the fact that the maximal Lyapunov exponent decreases with $\alpha$ (as shown in Fig. 4(a) and therefore perturbations of bigger and bigger amplitudes are required to destabilize the system for vanishingly pulse width. Consistently also the parameter $\beta$ associated to $P_{S}$ increases for increasing $\alpha$-values, as shown in the inset of Fig. 10 (b).

\section{Discussion}

We have investigated the dynamics and stability of current and fluctuation driven neural networks, the former (latter) have been realized as a purely excitatory (inhibitory) pulse coupled network of leaky integrate-and-fire (LIF) neurons with a sparse architecture. In particular, we considered random networks with a constant in-degree $K=20$ for any examined size. 
The excitatory network, despite being chaotic, reveals a low spiking variability. On the other hand, in the fluctuation driven case the variability is high for any considered pulse width and system size $(\mathrm{CV} \simeq 0.7-1.0)$. However, a different picture arises when studying the stability of infinitesimal perturbations: the system is chaotic for slow synapses and it becomes stable for sufficiently fast synaptic times $(\leq 4 \mathrm{~ms})$. Furthermore, a chaotic state for the inhibitory network is observable already at small connectivity $K \sim O\left(10^{1}\right)$ contradicting what reported in [16], where the authors affirmed that a large connectivity is a prerequisite to observe chaotic motion in these models.

The maximal Lyapunov exponent $\lambda$ tends towards an asymptotic value for increasing system sizes with a power-law scaling. The exponent $\eta$ associated to this scaling is different in the current (fluctuation) driven case, in particular $\eta \simeq 1(\eta \simeq 1 / 4)$ [30]. In the fluctuation driven situation the exponent is the same in the chaotic and stable phases. The origin of the observed scaling demands for new theoretical analysis, similar to the one developed for spatio-temporal chaotic systems [43].

Quite astonishingly even in the linearly stable regime an erratic evolution of the network is observable. A similar phenomenon has been already observed in several systems ranging from diffusively coupled chaotic maps to neural networks, and it has been identified as stable chaos [13]. In this context, finite amplitude perturbations are responsible for the erratic behavior observed in the system. In diffusively coupled systems this nonlinear instabilities has been characterized in terms of the propagation velocity of the information and of suitable Finite Size Lyapunov Exponents (FSLEs) 37, 41]. FSLEs have been previously employed in the context of fully coupled neural networks, where they revealed that the origin of the chaotic motion observed in two symmetrical coupled neural populations was due to collective chaos in the mean-field variables driving the single LIF neurons [28]. In the context of randomly coupled systems the concept of propagation velocity on a lattice looses his sense, while FSLEs reveal serious problems in their numerical implementation.

However, FSLEs clearly show also in our case that in the current driven case 
the observed instabilities have a purely linear origin, while in the fluctuation driven situation nonlinear mechanisms are present even when the system is chaotic. This analysis is confirmed by a novel indicator we have introduced, namely the local derivative $D(\Delta)$ of the averaged logarithmic distance $<\log \Delta>$ between the reference and the perturbed trajectory. This quantity suffers less than the FSLE the trial to trial fluctuations, since it is based on an averaged profile. For the fluctuation driven case this indicator is larger than the maximal Lyapunov exponent at finite amplitudes and this effect is present for all the examined system sizes. The position of the peak in $D(\Delta)$ seems not to be particularly influenced by the system size, while the peak itself broadens towards larger amplitudes for increasing $N$. Unfortunately, all these indications cannot tell us if the nonlinear mechanisms are prevailing on the linear ones, but just that the nonlinear effects are present. To measure the influence of linear versus nonlinear effects on the system dynamics, novel indicators are required, similar to linear and nonlinear information velocities for diffusively coupled systems [13].

As a final point we have studied nonlinear instabilities in linearly stable systems emerging in fluctuation driven inhibitory networks for sufficiently narrow postsynaptic currents. For the characterization of these instabilities we have employed the average finite amplitude growth rate $R_{T}\left(\Delta_{0}\right)$, measured after a finite time interval $T$, analogously to what what done in [34, 41], and the probability $P_{S}\left(\Delta_{0}\right)$ that an initial perturbation induces an exponential separation between the perturbed and the reference orbits, previoulsy introduced in [19]. Both these indicators reveal the existence of instabilities associated to finite perturbations, in particular the characteristic amplitude scales associated to these indicators vanish in the thermodynamic limit as $1 / N$. Thus suggesting that instabilities in these systems can occur even for infinitesimal perturbations in clear contradiction with the fact that these systems are linearly stable at any system size, as revealed by the Lyapunov analysis. This contradiction has lead Monteforte \& Wolf to conjecture in [19] that the thermodynamic limit and the limit of of vanishingly small perturbations do not commute in these models. Furtermore, we measure a logarithmic divergence with the system size of the 
peak height of $R_{T}\left(\Delta_{0}\right)$, suggesting that in the thermodynamic limit the value of these indicator will become infinite, similarly to what found in the high connectivity limit for a binary neuronal model in the balanced state [32] and for LIF with $\delta$-pulses in [19]. However, in our study the connectivity remains finite and small in the limit $N \rightarrow \infty$.

Our opinion, based on the comparison of the indicators $D(\Delta)$ and $R_{T}\left(\Delta_{0}\right)$ performed in a fluctuation driven chaotic situation, is that the above results can be due to the fact that the dynamics considered for the estimation of $R_{T}\left(\Delta_{0}\right)$ and $P_{S}\left(\Delta_{0}\right)$ do not take place on the attractor of the system. This because the indicators are estimated at short times, without allowing the perturbed dynamics to relax onto the attractor. The development of new indicators is required to analyze more in depth the phenomenon of Stable Chaos in randomly connected networks.

\section{Acknowledgments}

We thank A. Politi, S. Luccioli, and J. Berke for useful discussions. This work has been supported by the European Commission under the program "Marie Curie Network for Initial Training", through the project N. 289146, "Neural Engineering Transformative Technologies (NETT)". D. A.-G. would like also to acknowledge the partial support provided by "Departamento Adminsitrativo de Ciencia Tecnologia e Innovacion - Colciencias" through the program "Doctorados en el exterior - 2013".

\section{Appendix I}

The LIF model is usually expressed in physical units as follows 45$]$

$$
\tau_{m} \frac{d V}{d \hat{t}}=-\left(V(\hat{t})-V_{0}\right)+R_{m} I_{e x t}+\tau_{m} \hat{g} \hat{E}(\hat{t}) \quad,
$$

where $R_{m}$ is the specific mebrane resistance, $\tau_{m}$ the membrane time constant, and $V_{0}$ the resting potential. The transformation of the adimensional model (1) 
to (18) can be obtained by performing the following set of transformations

$$
\begin{array}{rrr}
V_{i}= & v_{i}\left(V_{t h}-V_{0}\right)+V_{0} \quad R_{m} I_{\text {ext }}= & a\left(V_{t h}-V_{0}\right)+V_{0} \\
\hat{g} & = & g\left(V_{t h}-V_{0}\right)+V_{0} \quad \hat{t}=\quad t \tau_{m},
\end{array}
$$

where $V_{t h}$ is the firing threshold value. Notice that $\hat{\alpha}=\alpha / \tau_{m}$ and $\hat{E}=E / \tau_{m}$ have the dimensionality of a frequency and $\hat{g}$ of a potential. Realistic values for the introduced parameters, are $\tau_{m}=20 \mathrm{~ms}, V_{0}=-60 \mathrm{mV}, V_{t h}=-50 \mathrm{mV}$ [46].

The postsynaptic current rise times $1 / \hat{\alpha}$ employed in this article range from 4 to $20 \mathrm{~ms}$ for inhibitory cells, while it is fixed to $2.22 \mathrm{~ms}$ for excitatory ones. Furthermore, the average neuronal firing rates are of order $\simeq 50 \mathrm{~Hz}(\simeq 6 \mathrm{~Hz})$ for excitatory (inhibitory) networks, which are quite reasonable values for pyramidal neurons (inter-neurons) of the cortex [47, 1, 48, 3]

\section{Bibliography}

[1] W. R. Softky, C. Koch, The highly irregular firing of cortical cells is inconsistent with temporal integration of random epsps, J. Neurosci. 13 (1) (1993) 334-350.

[2] G. R. Holt, W. R. Softky, C. Koch, R. J. Douglas, Comparison of discharge variability in vitro and in vivo in cat visual cortex neurons, J. Neurophysiol. 75 (5) (1996) 1806-1814.

[3] K. M. Stiefel, B. Englitz, T. J. Sejnowski, Origin of intrinsic irregular firing in cortical interneurons, P. Nat. Acad. Sci. USA 110 (19) (2013) 7886-7891.

[4] H. C. Tuckwell, Introduction to theoretical neurobiology: Volume 2, nonlinear and stochastic theories, Vol. 8, Cambridge University Press, 2005.

[5] L. F. Abbott, C. van Vreeswijk, Asynchronous states in networks of pulsecoupled oscillators, Phys. Rev. E 48 (2) (1993) 1483-1490.

[6] B. Haider, A. Duque, A. R. Hasenstaub, D. A. McCormick, Neocortical network activity in vivo is generated through a dynamic balance of excitation and inhibition, J. Neurosci. 26 (17) (2006) 4535-4545. 
[7] A. Renart, J. de la Rocha, P. Bartho, L. Hollender, N. Parga, A. Reyes, K. D. Harris, The asynchronous state in cortical circuits, Science 327 (5965) (2010) 587-590.

[8] A. Litwin-Kumar, B. Doiron, Slow dynamics and high variability in balanced cortical networks with clustered connections, Nature. Neurosci. 15 (11) (2012) 1498-1505.

[9] M. V. Tsodyks, T. Sejnowski, Rapid state switching in balanced cortical network models, Network-Comp. Neural. 6 (2) (1995) 111-124.

[10] N. Brunel, V. Hakim, Fast global oscillations in networks of integrate-andfire neurons with low firing rates, Neural. Comput. 11 (1) (1999) 1621-1671.

[11] T. P. Vogels, K. Rajan, L. Abbott, Neural networks dynamics, Annu. Rev. Neurosci. 28 (1) (2005) 357-376.

[12] N. Brunel, Dynamics of sparsely connected networks of excitatory and inhibitory spiking neurons, J. Comput. Neurosci. 8 (3) (2000) 183-208.

[13] A. Politi, A. Torcini, Stable chaos, in: Nonlinear Dynamics and Chaos: Advances and Perspectives, Springer, 2010, pp. 103-129.

[14] R. Zillmer, R. Livi, A. Politi, A. Torcini, Desynchronization in diluted neural networks, Phys. Rev. E 74 (3) (2006) 036203.

[15] S. Jahnke, R.-M. Memmesheimer, M. Timme, Stable irregular dynamics in complex neural networks, Phys. Rev. Lett. 100 (2008) 048102.

[16] R. Zillmer, N. Brunel, D. Hansel, Very long transients, irregular firing, and chaotic dynamics in networks of randomly connected inhibitory integrateand-fire neurons, Phys. Rev. E 79 (2009) 031909.

[17] S. Jahnke, R.-M. Memmesheimer, M. Timme, How chaotic is the balanced state?, Front. Comp. Neurosci. 3 (13). 
[18] S. Luccioli, A. Politi, Irregular Collective Behavior of Heterogeneous Neural Networks, Phys. Rev. Lett. 105 (15) (2010) 158104+.

[19] M. Monteforte, F. Wolf, Dynamic flux tubes form reservoirs of stability in neuronal circuits, Phys. Rev. X 2 (2012) 041007.

[20] A. Politi, R. Livi, G.-L. Oppo, R. Kapral, Unpredictable behaviour in stable systems, Europhys. Lett. 22 (8) (1993) 571.

[21] P. Tiesinga, J. V. José, T. J. Sejnowski, Comparison of current-driven and conductance-driven neocortical model neurons with hodgkin-huxley voltage-gated channels, Phys. Rev. E 62 (6) (2000) 8413.

[22] A. Renart, R. Moreno-Bote, X.-J. Wang, N. Parga, Mean-driven and fluctuation-driven persistent activity in recurrent networks, Neural. Comput. 19 (1) (2007) 1-46.

[23] M. London, A. Roth, L. Beeren, M. Häusser, P. E. Latham, Sensitivity to perturbations in vivo implies high noise and suggests rate coding in cortex, Nature. 466 (2010) 123-127.

[24] E. Aurell, G. Boffetta, A. Crisanti, G. Paladin, A. Vulpiani, Growth of noninfinitesimal perturbations in turbulence, Phys. Rev. Lett. 77 (1996) $1262-1265$.

[25] G. G. Turrigiano, K. R. Leslie, N. S. Desai, L. C. Rutherford, S. B. Nelson, Activity-dependent scaling of quantal amplitude in neocortical neurons, Nature 391 (6670) (1998) 892-896.

[26] G. G. Turrigiano, The self-tuning neuron: synaptic scaling of excitatory synapses, Cell 135 (3) (2008) 422-435.

[27] R. Zillmer, R. Livi, A. Politi, A. Torcini, Stability of the splay state in pulse-coupled networks, Phys. Rev. E 76 (2007) 046102.

[28] S. Olmi, R. Livi, A. Politi, A. Torcini, Collective oscillations in disordered neural networks., Phys. Rev. E 81 (4 Pt 2). 
[29] L. Tattini, S. Olmi, A. Torcini, Coherent periodic activity in excitatory erdos-renyi neural networks: the role of network connectivity, Chaos 22 (2) (2012) 023133.

[30] S. Luccioli, S. Olmi, A. Politi, A. Torcini, Collective dynamics in sparse networks, Phys. Rev. Lett. 109 (2012) 138103.

[31] M. Monteforte, F. Wolf, Dynamical entropy production in spiking neuron networks in the balanced state, Phys. Rev. Lett. 105 (26) (2010) 268104.

[32] C. van Vreeswijk, H. Sompolinsky, Chaos in neuronal networks with balanced excitatory and inhibitory activity, Science 274 (5293) (1996) 17241726.

[33] G. Benettin, L. Galgani, A. Giorgilli, J.-M. Strelcyn, Lyapunov characteristic exponents for smooth dynamical systems and for hamiltonian systems; a method for computing all of them. part 1: Theory, Meccanica 15 (1) (1980) 9-20.

[34] T. Letz, H. Kantz, Characterization of sensitivity to finite perturbations, Phys. Rev. E 61 (2000) 2533-2538.

[35] M. Cencini, F. Cecconi, A. Vulpiani, Chaos: From Simple Models to Complex Systems, Series on advances in statistical mechanics, World Scientific, 2010.

[36] A. Politi, Lyapunov exponent 8 (3) (2013) 2722.

[37] A. Torcini, P. Grassberger, A. Politi, Error propagation in extended chaotic systems, J. Phys. A-Math. Gen. 28 (16) (1995) 4533.

[38] T. Shibata, K. Kaneko, Collective chaos, Phys. Rev. Lett. 81 (1998) 41164119.

[39] M. Cencini, M. Falcioni, D. Vergni, A. Vulpiani, Macroscopic chaos in globally coupled maps, Physica D 130 (1) (1999) 58-72. 
[40] S. Olmi, A. Politi, A. Torcini, Collective chaos in pulse-coupled neural networks, Europhys. Lett. (2010) 60007+.

[41] M. Cencini, A. Torcini, Linear and nonlinear information flow in spatially extended systems, Phys. Rev. E 63 (2001) 056201.

[42] C. van Vreeswijk, Partial synchronization in populations of pulse-coupled oscillators, Phys. Rev. E 54 (5) (1996) 5522-5537.

[43] A. Pikovsky, A. Politi, Dynamic localization of lyapunov vectors in spacetime chaos, Nonlinearity 11 (4) (1998) 1049.

[44] A. Politi, S. Luccioli, Dynamics of networks of leaky integrate-and-fire neurons, in: Network Science: Complexity in Nature and Technology, Springer, London, 2010, p. 217.

[45] C. Koch, Biophysics of Computation: Information Processing in Single Neurons (Computational Neuroscience), 1st Edition, Oxford University Press, 1998.

[46] D. Sterratt, B. Graham, A. Gillies, D. Willshaw, Principles of Computational Modelling in Neuroscience, 1st Edition, Cambridge University Press, 2011.

[47] D. A. McCormick, B. W. Connors, J. W. Lighthall, D. A. Prince, Comparative electrophysiology of pyramidal and sparsely spiny stellate neurons of the neocortex, J. Neurophysiol. 54 (4) (1985) 782-806.

[48] A. Destexhe, M. Rudolph, J.-M. Fellous, T. J. Sejnowski, Fluctuating synaptic conductances recreate in vivo-like activity in neocortical neurons, Neuroscience. 107 (1) (2001) 13-24. 